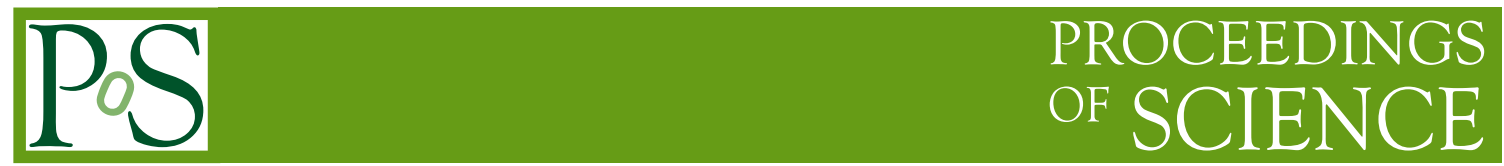

\title{
QCD description of ATLAS jet veto data
}

\section{Dominik Werder*}

Uppsala University, Box 516, 75120 Uppsala, Sweden

E-mail: dominik.werderephysics.uu.se

Dijet production with a veto on the additional jets between the dijet system is studied by using an approach by Banfi, Marchesini and Smye, which resums both the Sudakov logarithms as well as the non-global logarithms due to wide angle gluon emissions to leading log accuracy. The prediction is compared to a recent measurement by ATLAS and we show that a good description of the data is obtained.

The European Physical Society Conference on High Energy Physics - EPS-HEP2013 18-24 July 2013

Stockholm, Sweden

${ }^{*}$ Speaker. 


\section{Introduction}

While jet-gap-jet events have been previously studied $[1,2,3,4,5]$, in this work we consider [6] the recent measurement by ATLAS [7] of the fraction of di-jet events with an additional veto on jets in the inter-jet region

$$
\mathcal{R}\left(\Delta y, p_{T}\right) \equiv \frac{d \sigma^{\text {veto }}}{d \Delta y d^{2} p_{T}} / \frac{d \sigma^{\text {incl }}}{d \Delta y d^{2} p_{T}}
$$

where $p_{T}$ denotes the average transverse momentum $p_{T}=\left(p_{1 T}+p_{2 T}\right) / 2$ and $\Delta y$ the rapidity difference between the boundary jets, for which the $p_{T}$ of additional jets in the inter-jet region is below a threshold $E_{\text {out }}$, but not necessarily zero. This observable is for large separation $\Delta y$ and small $E_{\text {out }}$ not only sensitive to BFKL physics, but also to large angle gluon emissions, which makes it interesting for studies of perturbative QCD. The di-jet system in [7] is defined in two different ways, one being the highest- $p_{T}$ pair and the other the most forward/backward pair in rapidity. For the veto cross section $\sigma^{\text {veto }}$ one requires that no additional jet having $p_{T}>E_{\text {out }}$ is reconstructed in the rapidity interval of the chosen boundary jets. $E_{\text {out }}$ is a free parameter with $E_{\text {out }}=20 \mathrm{GeV}$ the smallest value which was considered in the measurement. Jets are reconstructed using an anti- $k_{t}$ algorithm [8] with the radius parameter $R=0.6$. The precise choice of $E_{\text {out }}$ affects the sensitivity to possible observables as we will see. We note that predictions from HeRWIG and PYTHIA $[9,10]$, even though not optimized for this observable [11], describe the data reasonably well, while fixed order calculations like ALPGEN and PowHEg [12, 13] undershoot the data significantly and are also not [6] expected to give stable result in the interesting region $\Delta y>2$. The inclusion of BFKL logarithms due to the possibly large $\Delta y$ separation of the di-jet system does not improve the description [14]. Also, contributions from non-perturbative QCD effects are expected to be negligible due to the choice of scales. The reason for the observed discrepancy may indeed lie in the angular-ordered parton showers, whereas the radiation in the inter-jet region of the system at hand is ordered in transverse momentum. The resummation in this case results in potentially large logarithms of the type $\left(\alpha_{s} \ln p_{T} / E_{\text {out }}\right)^{n}$, possibly further enhanced by large rapidity separation $\left(\alpha_{s} \Delta y \ln p_{T} / E_{\text {out }}\right)^{n}$. One class of logarithms comes from the emissions from the primary parton, giving rise to the well known Sudakov type logarithms $[15,16]$. A second class of terms comes from the recursive emissions from secondary gluons [17] giving rise to the so-called non-global logarithms which can only in the large- $N_{c}$ limit be resummed. Previous works already included non-global emissions by the introduction of a survival factor, while a resummation of these relevant logarithms to all orders is expected to result in a more accurate description of the data measured by ATLAS.

\section{Jet veto cross section using BMS formalism}

Using the approach by [18] the problem of recursive emissions in the presence of a veto threshold is cast into a differential equation which can be solved numerically $[19,6]$. To describe the jet veto observable, we consider the leading order one gluon color-octet exchange channel. For the geometry at hand, we define two cones centered around the beam axis with their opening angles chosen such that they just enclose the two boundary jets resulting from a color-singlet $q \bar{q}$ pair coming from the hard interaction. The probability that the energy radiated outside of the cones is less 


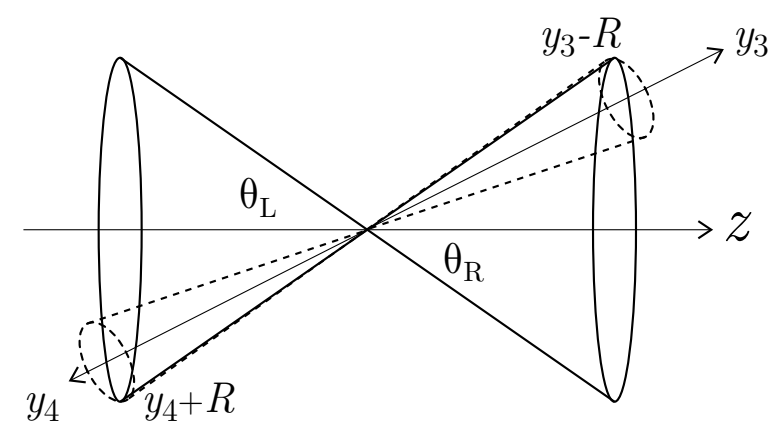

Figure 1: Geometry for the dijet inter-jet region with $y=\ln \cot (\theta / 2)$.

than the threshold $E_{\text {out }}$ is denoted by $P_{\tau}\left(\theta_{R}, \theta_{L}, \Omega_{3}, \Omega_{4}\right)$ with $\Omega=(y, \varphi)$ being the direction of the jets. $P_{\tau}$ will not depend on the azimuthal angle $\varphi$ because we have $\varphi_{4}-\varphi_{3}=\pi$. Defining

$$
\begin{aligned}
\alpha_{s}\left(p_{T}\right) & =\pi /\left(2 b N_{c} \ln \frac{p_{T}}{\Lambda_{\mathrm{QCD}}}\right) \\
b & =\frac{11 N_{c}-2 n_{f}}{12 N_{c}}
\end{aligned}
$$

the evolution variable $\tau$ of $P_{\tau}$ for the case of running $\alpha_{s}$ reads

$$
\tau=\int_{E_{\text {out }}}^{p_{T}} \frac{d k_{T}}{k T} \frac{\alpha_{s}\left(k_{T}\right) N_{c}}{\pi}=\frac{1}{2 b} \ln \left(\alpha_{s}\left(E_{\text {out }}\right) / \alpha_{s}\left(p_{T}\right)\right)=\frac{1}{2 b} \ln \frac{\ln \left(p_{T} / \Lambda_{\mathrm{QCD}}\right)}{\ln \left(E_{\mathrm{out}} / \Lambda_{\mathrm{QCD}}\right)}
$$

while the evolution equation itself can be written as

$$
\begin{aligned}
\partial_{\tau} P_{\tau}\left(\Omega_{\alpha}, \Omega_{\beta}\right)= & -\int_{C_{\text {out }}} \frac{d^{2} \Omega_{\gamma}}{4 \pi} \frac{1-\cos \theta_{\alpha \beta}}{\left(1-\cos \theta_{\alpha \gamma}\right)\left(1-\cos \theta_{\gamma \beta}\right)} P_{\tau}\left(\Omega_{\alpha}, \Omega_{\beta}\right) \\
& +\int_{C_{\text {in }}} \frac{d^{2} \Omega_{\gamma}}{4 \pi} \frac{1-\cos \theta_{\alpha \beta}}{\left(1-\cos \theta_{\alpha \gamma}\right)\left(1-\cos \theta_{\gamma \beta}\right)}\left(P_{\tau}\left(\Omega_{\alpha}, \Omega_{\gamma}\right) P_{\tau}\left(\Omega_{\gamma}, \Omega_{\beta}\right)-P_{\tau}\left(\Omega_{\alpha}, \Omega_{\beta}\right)\right)
\end{aligned}
$$

resumming both Sudakov and non-global logarithms. At zeroth order $P_{\tau}=1$ due to no additional emissions. $P_{\tau}$ is also invariant under Lorentz boosts and therefore

$$
P_{\tau}\left(y_{R}, y_{L}, y_{3}, y_{4}\right)=P_{\tau}\left(\frac{y_{R}-y_{L}}{2},-\frac{y_{R}-y_{L}}{2}, y_{3}-\frac{y_{R}+y_{L}}{2}, y_{4}-\frac{y_{R}+y_{L}}{2}\right) .
$$

In the large $N_{c}$ limit, the contributions to parton showers may be decomposed into radiation from dipole systems. We consider the tree-level $2 \rightarrow 2$ matrix elements with their cross sections, e.g. for $q q^{\prime} \rightarrow q q^{\prime}$

$$
\frac{d \sigma_{q q^{\prime}}}{d \hat{t}}=\frac{1}{16 \pi \hat{s}^{2}} h^{A}(\hat{s}, \hat{t}, \hat{u})=\frac{1}{16 \pi \hat{s}^{2}} g^{4} \frac{C_{F}}{N_{c}}\left(\frac{s^{2}+u^{2}}{t^{2}}\right)
$$

where the color flows in large- $N_{c}$ like $1 \rightarrow 4$ and $2 \rightarrow 3$ which results in the two dipoles (14) and (23). Incorporating the probabilities $P_{\tau}$ for radiations in the vetoed inter-jet region one obtains

$$
\begin{array}{r}
\frac{d \sigma_{q q^{\prime}}^{\text {veto }}}{d \hat{t}}=\frac{1}{16 \pi \hat{s}^{2}}\left(h^{A}(\hat{s}, \hat{t}, \hat{u}) P_{\tau}\left(y_{3}-R, y_{4}+R, \infty, y_{4}\right) P_{\tau}\left(y_{3}-R, y_{4}+R, y_{3},-\infty\right)\right. \\
\left.+h^{A}(\hat{s}, \hat{u}, \hat{t}) P_{\tau}\left(y_{3}-R, y_{4}+R, \infty, y_{3}\right) P_{\tau}\left(y_{3}-R, y_{4}+R, y_{4},-\infty\right)\right)
\end{array}
$$


Because of the boost invariance of $P_{\tau}$ we only need the evaluate in the center of mass frame:

$$
\begin{gathered}
P_{\tau}\left(y_{3}-R, y_{4}+R, \infty, y_{4}\right)=P_{\tau}\left(\frac{\Delta y}{2}-R,-\frac{\Delta y}{2}+R, \infty,-\frac{\Delta y}{2}\right) \equiv P_{14}(\tau, \Delta y) \\
P_{\tau}\left(y_{3}-R, y_{4}+R, y_{3},-\infty\right)=P_{\tau}\left(\frac{\Delta y}{2}-R,-\frac{\Delta y}{2}+R, \frac{\Delta y}{2},-\infty\right) \equiv P_{23}(\tau, \Delta y) \\
P_{\tau}\left(y_{3}-R, y_{4}+R, \infty, y_{3}\right)=P_{\tau}\left(\frac{\Delta y}{2}-R,-\frac{\Delta y}{2}+R, \infty, \frac{\Delta y}{2}\right) \equiv P_{13}(\tau, \Delta y) \\
P_{\tau}\left(y_{3}-R, y_{4}+R, y_{4},-\infty\right)=P_{\tau}\left(\frac{\Delta y}{2}-R,-\frac{\Delta y}{2}+R,-\frac{\Delta y}{2},-\infty\right) \equiv P_{24}(\tau, \Delta y)
\end{gathered}
$$

Since $P_{14}=P_{23}$ and $P_{13}=P_{24}$, the cross section takes the simpler form

$$
\begin{aligned}
\frac{d \sigma_{q q^{\prime}}^{\text {veto }}}{d \hat{t}} & =\frac{1}{16 \pi \hat{s}^{2}}\left(h^{A}(\hat{s}, \hat{t}, \hat{u}) P_{14} P_{23}+h^{A}(\hat{s}, \hat{u}, \hat{t}) P_{13} P_{24}\right) \\
& =\frac{1}{16 \pi \hat{s}^{2}}\left(h^{A}(\hat{s}, \hat{t}, \hat{u}) P_{14}^{2}+h^{A}(\hat{s}, \hat{u}, \hat{t}) P_{13}^{2}\right)
\end{aligned}
$$

The vetoed cross sections of the other possible partonic subprocesses can be obtained in a similar way [6] and yield via convolution with the proton parton density functions the hadronic vetoed cross section

$$
\frac{d \sigma^{\text {veto }}}{d \Delta y d^{2} p_{T}}=\sum_{i j}^{q, \bar{q}, g} \int_{Y_{\min }}^{Y_{\max }} d Y x_{1} f_{i}\left(x_{1}, p_{T}\right) x_{2} f_{j}\left(x_{2}, p_{T}\right) \frac{1}{\pi} \frac{d \sigma_{i j}^{\text {veto }}}{d \hat{t}}
$$

where $Y=\frac{y_{3}+y_{4}}{2}$ and $|Y|<4.4$ for the ATLAS measurement.

\section{Comparison with ATLAS data}

The vetoed cross section $\sigma$ as computed before is divided by the inclusive $\sigma$ i.e. with $P_{\tau}=1$ to yield the veto fraction $\mathcal{R}$. Fig. 2 shows the comparison with ATLAS data for the dependence of $\mathcal{R}$ on the jet rapidity separation $\Delta y$ for different bins in jet $p_{T}$. We note that the model is in quite good agreement with the data for the forward/backward selection. Fig. 2 also illustrates the theoretical uncertainties coming from the factorization and renormalization scale uncertainty, obtained by varying the scale for the parton density and by varying $\alpha_{s}$ in the amplitudes and in the definition of $\tau$. The uncertainty on the subleading logarithms is estimated by varying the upper limit of the integrand in the definition of $\tau$. Then, Fig. 3 shows the dependence on the veto threshold $E_{\text {out }}$. Since a large scale separation between the jet $p_{T}$ and the threshold $E_{\text {out }}$ is assumed in the calcuation, the agreement is best for small values of $E_{\text {out }}$.

\section{Conclusions and Outlook}

We have investigated [6] the resummation of the Sudakov and the non-global logarithms induced by large angle soft gluon emissions in the context of the jet veto cross section. The ATLAS measurement corresponding to the forward/backward selection is described well by tree-level QCD together with the jet veto probability as given in the BMS approach. We have also seen that the data 




Figure 2: The fraction of the vetoed cross section is compared against the ATLAS data for $E_{\text {out }}=20 \mathrm{GeV}$ in different regions of average jet $p_{T}$. Number of active flavors is $n_{f}=5$ and $\alpha_{s}$ is fixed as $\alpha_{s}\left(M_{Z}\right)=0.12$. The green (inner) error band corresponds to the factorization and renormalization scale uncertainties. The yellow (outer) band includes the uncertainty from the subleading logarithms as well. ATLAS data for both selection criteria of the boundary jets is shown. The upper points correspond to the leading $p_{T}$ selection whereas the lower points correspond to the most max/min rapidity selection.

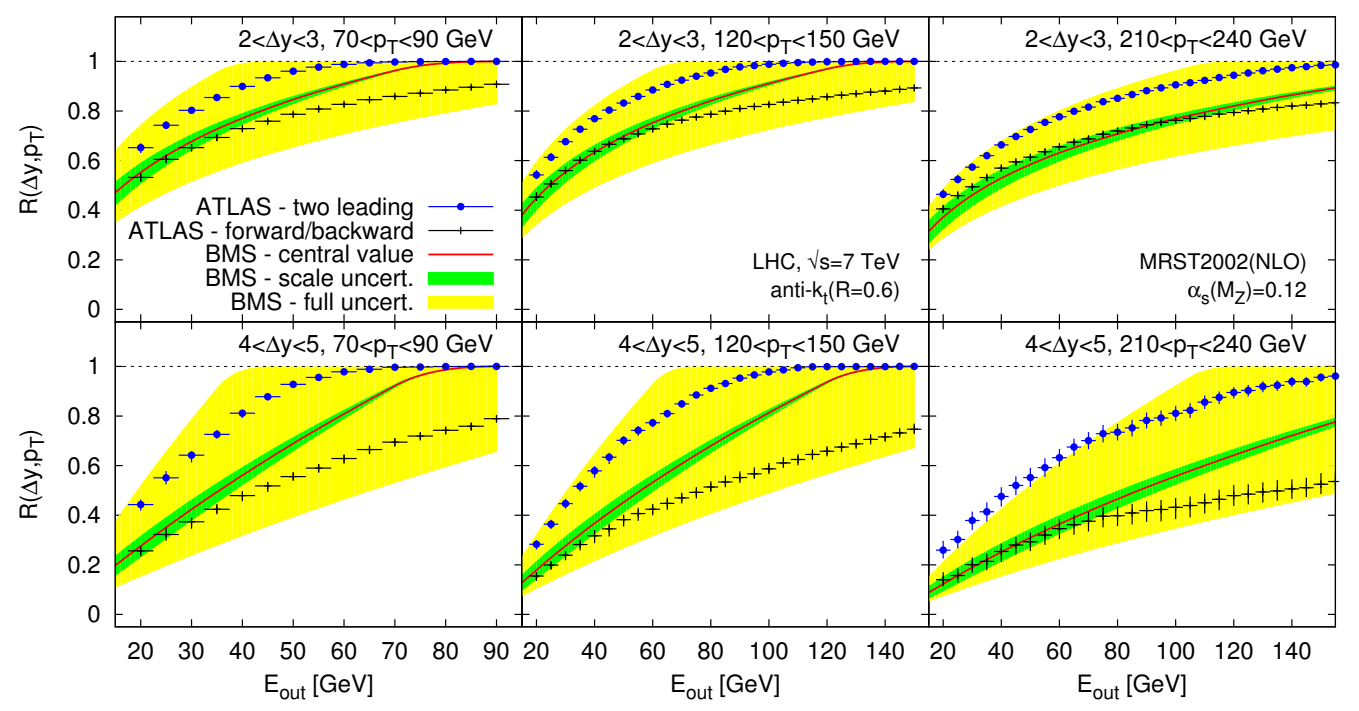

Figure 3: Same conditions as in Fig. 2 but varying the veto threshold $E_{\text {out }}$ as well. The agreement with data is best for a large separation between $E_{\text {out }}$ and $p_{T}$ as expected. 
are well described by the color octet contribution alone, without the need for additional BFKL-like contributions which is expected given the scale of $E_{\text {out }}$. Due to the still large theoretical uncertainties it is not possible to fully distinguish between the two selection methods used by the experiment. Inclusion of subleading logarithms and $1 / N_{c}$ corrections could improve this result.

\section{References}

[1] B. Abbott et al. [D0 Collaboration], Probing hard color-singlet exchange in p $\bar{p}$ collisions at $\sqrt{s}=630 \mathrm{GeV}$ and $1800 \mathrm{GeV}$, Phys. Lett. B 440, 189 (1998). [arXiv: hep-ex/9809016]

[2] F. Abe et al. [CDF Collaboration], Di-jet production by color-singlet exchange at the Fermilab Tevatron, Phys. Rev. Lett. 80, 1156 (1998).

[3] H. Navelet, R. B. Peschanski, C. Royon and S. Wallon, Proton structure functions in the dipole picture of BFKL dynamics, Phys. Lett. B 385, 357 (1996) [hep-ph/9605389].

[4] O. Kepka, C. Marquet and C. Royon, Gaps between jets in hadronic collisions, Phys. Rev. D 83, 034036 (2011) [arXiv:1012.3849 [hep-ph]].

[5] C. Marquet, C. Royon, M. Trzebiński and R. Žlebčík, Gaps between jets in double-Pomeron-exchange processes at the LHC, Phys. Rev. D 87, no. 3, 034010 (2013) [arXiv:1212.2059 [hep-ph] ].

[6] Y. Hatta, C. Marquet, C. Royon, G. Soyez, T. Ueda and D. Werder, A QCD description of the ATLAS jet veto measurement, Phys. Rev. D 87, 054016 (2013) [arXiv:1301.1910 [hep-ph] ].

[7] G. Aad et al. [ATLAS Collaboration], Measurement of dijet production with a veto on additional central jet activity in pp collisions at sqrt( $(s)=7 \mathrm{TeV}$ using the ATLAS detector, JHEP 1109, 053 (2011). [arXiv:1107.1641 [hep-ex]]

[8] M. Cacciari, G. P. Salam and G. Soyez, The anti-kt jet clustering algorithm, JHEP 0804 (2008) 063. [arXiv:0802.1189]

[9] M. Bahr, S. Gieseke, M. A. Gigg, D. Grellscheid, K. Hamilton, O. Latunde-Dada, S. Platzer and P. Richardson et al., Herwig++ Physics and Manual, Eur. Phys. J. C 58 (2008) 639. [arXiv:0803.0883 [hep-ph]]

[10] T. Sjostrand, S. Mrenna and P. Skands, Pythia 6.4 physics and manual, JHEP 0605 (2006) 026. [arXiv:hep-ph/0603175]

[11] A. Banfi, G. Corcella and M. Dasgupta, Angular ordering and parton showers for non-global QCD observables, JHEP 0703, 050 (2007) [hep-ph / 0612282 ].

[12] M. L. Mangano, M. Moretti, F. Piccinini, R. Pittau and A. D. Polosa, ALPGEN, a generator for hard multiparton processes in hadronic collisions, JHEP 0307 (2003) 001. [hep-ph/ 0206293 ]

[13] S. Alioli, P. Nason, C. Oleari and E. Re, A general framework for implementing NLO calculations in shower Monte Carlo programs: the POWHEG BOX, JHEP 1006 (2010) 043. [arXiv: 1002.2581 [hep-ph] ]

[14] J. R. Andersen and J. M. Smillie, High Energy Description of Processes with Multiple Hard Jets, Nucl. Phys. Proc. Suppl. 205-206 (2010) 205 [arXiv: 1007.4449 [hep-ph] ]; JHEP 1106 (2011) 010 [arXiv:1101.5394 [hep-ph] ].

[15] G. Oderda and G. F. Sterman, Energy and color flow in dijet rapidity gaps, Phys. Rev. Lett. 81, 3591 (1998) [hep-ph/9806530]. 
[16] J. Forshaw, J. Keates and S. Marzani, Jet vetoing at the LHC, JHEP 0907, 023 (2009) [arXiv:0905.1350 [hep-ph]].

[17] M. Dasgupta and G. P. Salam, Resummation of nonglobal QCD observables, Phys. Lett. B 512, 323 (2001) [hep-ph/0104277].

[18] A. Banfi, G. Marchesini and G. Smye, Away from jet energy flow, JHEP 0208, 006 (2002). [arXiv:hep-ph/0206076]

[19] Y. Hatta and T. Ueda, Jet energy flow at the LHC, Phys. Rev. D 80, 074018 (2009). [arXiv:0909.0056 [hep-ph]] 